\title{
ECCDIA: an interactive web tool for the comprehensive analysis of clinical and survival data of esophageal cancer patients
}

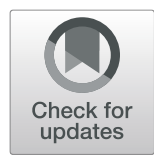

Jingcheng Yang ${ }^{1 \dagger}$, Jun Shang ${ }^{1 \dagger}$, Qian Song ${ }^{1 \dagger}$, Zuyi Yang ${ }^{2}$, Jianing Chen ${ }^{3}$, Ying $Y_{u^{1,4,5^{*}}}$ and Leming Shi ${ }^{1,4,5^{*}}$

\begin{abstract}
Background: Esophageal cancer (EC) is considered as one of the deadliest malignancies with respect to incidence and mortality rate, and numerous risk factors may affect the prognosis of EC patients. For better understanding of the risk factors associated with the onset and prognosis of this malignancy, we develop an interactive web-based tool for the convenient analysis of clinical and survival characteristics of EC patients.

Methods: The clinical data were obtained from The Surveillance, Epidemiology, and End Results (SEER) database. Seven analysis and visualization modules were built with Shiny.

Results: The Esophageal Cancer Clinical Data Interactive Analysis (ECCDIA, http://webapps.3steps.cn/ECCDIA) was developed to provide basic data analysis, visualization, survival analysis, and nomogram of the overall group and subgroups of 77,273 EC patients recorded in SEER. The basic data analysis modules contained distribution analysis of clinical factor ratios, Sankey plot analysis for relationships between clinical factors, and a map for visualizing the distribution of clinical factors. The survival analysis included Kaplan-Meier (K-M) analysis and Cox analysis for different subgroups of EC patients. The nomogram module enabled clinicians to precisely predict the survival probability of different subgroups of EC patients.
\end{abstract}

Conclusion: ECCDIA provides clinicians with an interactive prediction and visualization tool for visualizing invaluable clinical and prognostic information of individual EC patients, further providing useful information for better understanding of esophageal cancer.

Keywords: Esophageal cancer, Clinical data mining, Survival analysis, Nomogram, SEER

\section{Background}

Esophageal cancer (EC) is considered as one of the most deadly malignancies with respect to incidence and mortality rate [1,2]. Globally, EC was ranked the seventh for the incidence rate and the sixth for the mortality rate in 2018 [2]. Approximately 17,650 new cases of EC are expected to occur and 16,080 patients are predicted to die

\footnotetext{
* Correspondence: ying_yu@fudan.edu.cn; lemingshi@fudan.edu.cn ${ }^{\dagger}$ Jingcheng Yang, Jun Shang and Qian Song are joint first authors. 'State Key Laboratory of Genetic Engineering, School of Life Sciences and Shanghai Cancer Hospital/Cancer Institute, Fudan University, Shanghai 200438, China

Full list of author information is available at the end of the article
}

from esophageal cancer in the United States in 2019 [1]. Previous studies have revealed numerous risk factors that may affect the prognosis of EC patients [3-6]. Nevertheless, these studies have been outdated and unable to provide an interactive and continuously updated result for researchers and physicians.

Population-based studies have been widely utilized to predict patients' survival outcomes and have played a significant role for clinical decision makers and for the recommendations of guidelines [7]. With the rise of interactive data analysis, there have been many tools to help us understand the molecular characteristics of EC,

(c) The Author(s). 2020 Open Access This article is licensed under a Creative Commons Attribution 4.0 International License, which permits use, sharing, adaptation, distribution and reproduction in any medium or format, as long as you give appropriate credit to the original author(s) and the source, provide a link to the Creative Commons licence, and indicate if changes were made. The images or other third party material in this article are included in the article's Creative Commons licence, unless indicated otherwise in a credit line to the material. If material is not included in the article's Creative Commons licence and your intended use is not permitted by statutory regulation or exceeds the permitted use, you will need to obtain permission directly from the copyright holder. To view a copy of this licence, visit http://creativecommons.org/licenses/by/4.0/. The Creative Commons Public Domain Dedication waiver (http://creativecommons.org/publicdomain/zero/1.0/) applies to the data made available in this article, unless otherwise stated in a credit line to the data. 
but there is still a lack of effective interactive web tools based on population statistics data of EC to help us fully understand the risk factors associated with the onset and prognosis of this malignancy. The Surveillance, Epidemiology, and End Results (SEER) database is an authoritative source for cancer statistics with comprehensive clinical and pathological information of cancer cases reported in the United States [8]. Based on SEER data, many studies have been conducted to explore the epidemic, clinicopathologic and prognostic characteristics of $\mathrm{EC}$, and to examine numerous risk factors that might be affected [3-6], but no study provides an interactive visual analysis of all the characteristics of EC data based on the SEER database [9]. Moreover, because SEER data are updated annually, the value of statistical results published from these studies using "outdated data" is somehow limited, resulting in limited usage of these precious data. Clinicians who would like to obtain valuable and updated information on EC prognosis may find it hard to navigate the rich data in SEER in whichever way they want.

Herein, we developed a powerful user-friendly webbased platform called Esophageal Cancer Clinical Data Interactive Analysis (ECCDIA) using data on 77,273 EC patients in the SEER Program from 1975 to 2018. ECCDIA is able to provide on-line statistical analysis tools, including clinical factor ratio distribution by year, the Sankey plots presenting relationships between different clinical factors, the survival rate analysis, Kaplan-Meier (K-M) analysis, Cox analysis, and nomograms illustrating prediction of survival probability across subgroups. ECCDIA is an efficient and user-friendly tool to assist researchers and clinicians to understand esophageal cancer using interactive analysis tools which can help users quickly explore data using different visualization approaches. ECCDIA is freely accessible and available at http://webapps.3steps.cn/ECCDIA/.

\section{Methods}

\section{Patient data}

Patient data were retrieved from the SEER*Stat Version 8.3.5 database named Incidence-SEER 18 Regs Custom Data (with additional treatment fields), Nov 2018 Sub (1973-2016 varying) by using the case-listing session. The International Classification of Diseases for Oncology (ICD-O-3) was utilized to identify patients with esophageal squamous cell carcinoma (ESCC) (ICD-O-3 histologic type: 8050-8089) and esophageal adenocarcinoma (EAD) (ICD-O-3 histologic type: 8140-8389) [10]. The ICD-O-3 site codes for EC were C15.0, 15.1, 15.2, 15.3, $15.4,15.5,15.8$, and 15.9. As the SEER database is a public one, there is no personal identification information for patients. Patients with diagnosed confirmation of positive histology and those of active follow up were included for analysis. Patients with unknown survival data were excluded. There were 77,273 patients for overall survival (OS) and 52,206 patients for cancer specific death (CSS).

\section{Construction of analysis modules}

ECCDIA is a web-based tool constructed with the Shiny framework. It contained seven interactive analysis modules written with $\mathrm{R}$ language (Fig. 1). Basic charts, such as bar plot, Sankey plot, line plot and map, were constructed with Plotly [11]. Cox and survival analysis were performed with $\mathrm{R}$ packages survival (v2.42-6) [12] and survminer (v0.4.3) [13]. Nomogram was constructed with R package rms (v5.1-2) [14].

\section{Results}

\section{Data summary}

Patients with unknown survival data were excluded. There were 77,273 patients including 58,668 males and 18,605 females for overall survival (OS) and 52,206 patients for cancer specific death (CSS). The histological type of 40,683 cases is esophageal adenocarcinoma (EAD), and the other 36,590 cases is esophageal squamous cell carcinoma (ESCC). Based on the 7th edition of AJCC, 3625, 3304, 5018 and 6287 patients were in stages I, II, III and IV, respectively.

\section{Modules of ECCDIA}

ECCDIA is a modular interactive tool which mainly contains seven capabilities, including "Clinical Ratio" that analyzes clinical factor ratio distribution by year, "Sankey Plot" that demonstrates the relationship of frequency distribution between different clinical factors, "Survival Rate" that exhibits the changes of survival rate for clinical factors by year, "K-M Analysis" that displays survival curves of OS and CSS for clinical factors, "Cox Analysis" that exhibits univariate and multivariate analysis of OS

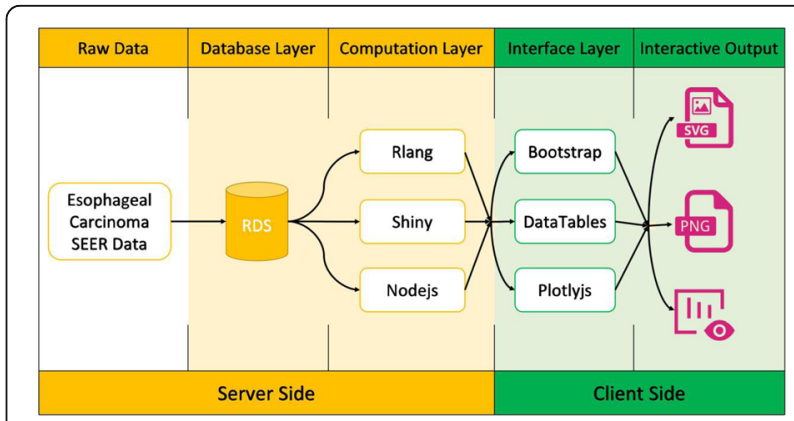

Fig. 1 Framework of ECCDIA. Schema describing data processing and data visualization for the ECCDIA. Raw data contains information for 77,273 esophageal cancer (EC) patients. Computation layer contains survival analysis, Cox analysis and nomogram modules. Interactive output contains basic charts, such as bar plot, Sankey plot, line plot and map, etc. 
and CSS for different subgroups of EC patients, "Nomogram" that predicts survival outcome for different subgroups of EC patients, and "Map" that exhibits the distribution of clinical factors in the form of a map of the United States (Fig. 2).

\section{Basic data analysis}

The first module of ECCDIA aims to find out the trend of different clinical factor ratio distribution by year. As exhibited in Figure S1A, the incidence of EAD increased, while the incidence of ESCC decreased by year. To further investigate whether this trend existed in the subgroup of EC patients, the different subgroups of data could be chosen. Interestingly, we found that male and white patients had a similar trend as the whole group, but the female, black and API (Asian or Pacific Islander) patients did not demonstrate a significant trend change (Figure S1B-F). Additional fascinating results can be found by users using this module of ECCDIA.

The flows of patients' module are to provide users with a convenient and intuitive interface for the correlation of different clinical factors. As demonstrated in Figure S2, most of the white patients were ESCC in 1975. In 1993, the proportion of EAD and ESCC was almost equal in white patients, whereas the majority of white patients were EAD in 2016. Users can perform interactive analysis in this module to find what they may be interested in. The last module of ECCDIA provides users with a map of the United States to show the distribution of clinical factors by state.

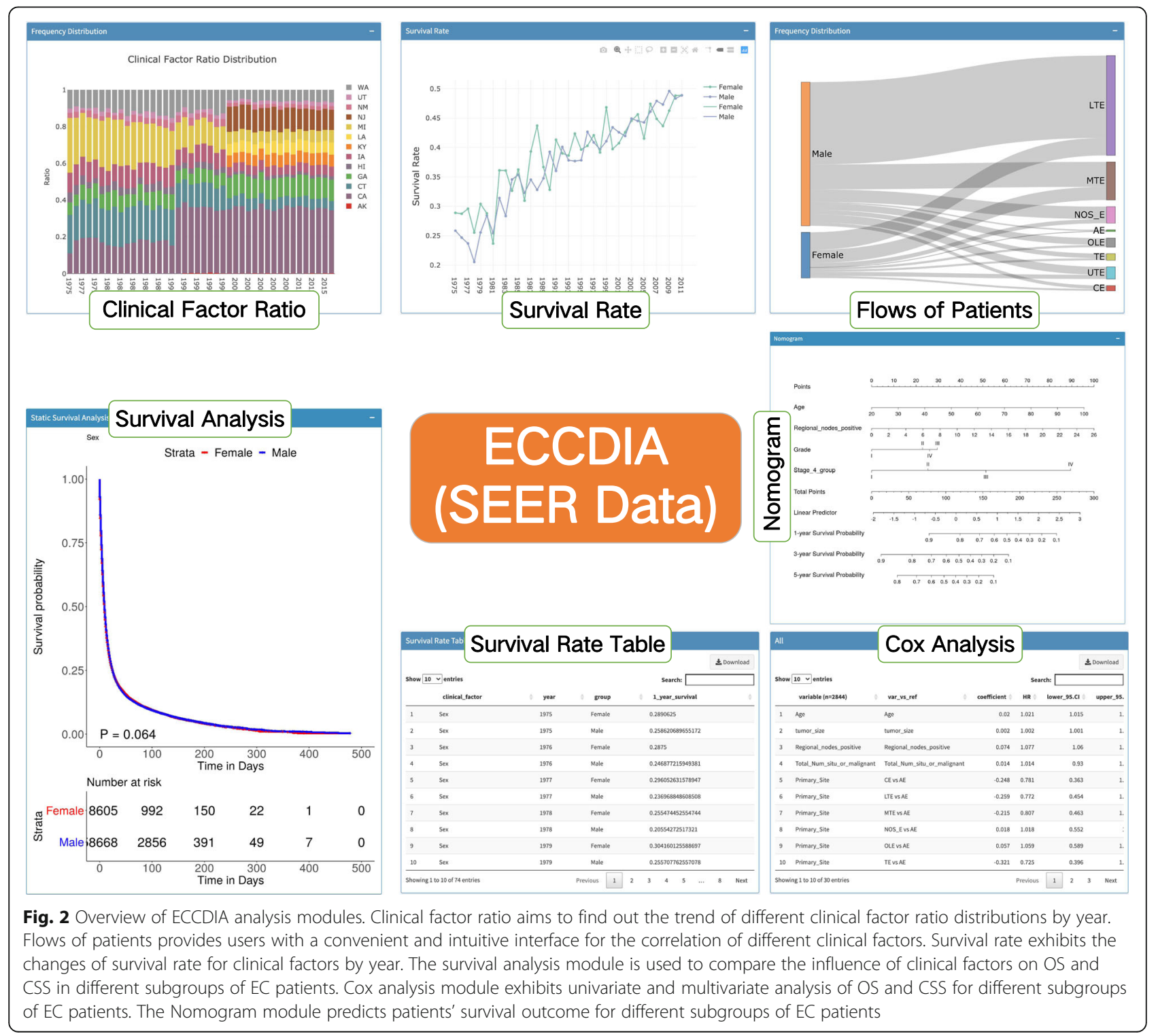




\section{Survival analysis}

The survival analysis mainly contains three modules, including survival rate, K-M analysis, and Cox analysis. The survival rate module has the ability to show EC patients survival rate fluctuation by year. We showed in Figures S3A and S3B how to perform survival analysis quickly and easily. ECCDIA allows us to quickly find that there were clear differences in survival among different histopathological types and ethnicities. In Figure S4A, before 1989, there exhibited an obvious fluctuation of survival rate between EAD and ESCC. Nevertheless, EAD patients tended to have a consistently higher survival rate than ESCC patients after 1989.

The K-M analysis module provides intuitive figures for users who would like to compare the impact of clinical factors on OS and CSS in different subgroups of EC patients. For instance, Figure S4B-D exhibited a comparison of the impact of histologic types on patients' OS. Regardless of male or female subgroup, EAD patients had a much better OS than ESCC patients.

The Cox analysis module demonstrates tables for the results of univariate and multivariate analysis of OS and CSS for different subgroups of EC patients. This module is also user-friendly and provides users with interactive tables.

\section{Nomogram}

The nomogram module can precisely predict 1-year, 3year, and 5-year survival probabilities of OS and CSS for all patients, ESCC patients, EAD patients, stages I-II patients, stages III-IV patients, and patients undergoing surgery plus chemotherapy and radiation therapy. For instance, for an EC patient who was 20 years old and had three positive regional nodes with grade I and stage
I, the 1-year, 3-year, and 5-year survival probabilities were 96.82, 90.19, and 86.25\%, respectively (Figure S5). At the same time, we show that there is a good agreement between the nomogram-based survival rates and the actual survival rate by using calibration plots (Figure S5C). Furthermore, the agreement between predicted and observed 1, 3, 5-year survival rates shown with calibration curves was verified with clinical data associated with the TCGA esophageal cancer data set (Figure S6).

\section{Map}

"Map", the last module of ECCDIA, provides users with a map of the United States to show the distribution of clinical factors by state. The distribution of the different survival rates is displayed in Data exploration and Interactive map sections. These functions can easily visualize the survival rate in different states for the EC patients (Table 1).

\section{Discussion}

This study provides an interactive web tool that analyzes rich clinical and prognostic data of EC patients from the SEER database. Our tool is able to provide clinicians and clinical decision makers with useful information to make suitable treatment plan for EC patients with no need to refer to a large number of research papers.

The SEER database has such a large collection of cancer patients' clinicopathologic and prognostic data so that it holds a great potential to conduct robust statistical mining to gain the most powerful and reliable survival prediction for cancer patients. However, previously published researches using the SEER database present analyses in only one aspect or the other and do

Table 1 The survival rates of esophageal cancer patients in different states

\begin{tabular}{|c|c|c|c|c|c|c|c|c|}
\hline State & $\begin{array}{l}\text { Num_- } \\
\text { Patients }\end{array}$ & $\begin{array}{l}\text { Average_ } \\
\text { age }\end{array}$ & $\begin{array}{l}\text { Average_tumor_ } \\
\text { size }(\mathrm{mm})\end{array}$ & $\begin{array}{l}\text { 1-year survival } \\
\text { rate }\end{array}$ & $\begin{array}{l}\text { 2-year survival } \\
\text { rate }\end{array}$ & $\begin{array}{l}\text { 3-year survival } \\
\text { rate }\end{array}$ & $\begin{array}{l}\text { 4-year survival } \\
\text { rate }\end{array}$ & $\begin{array}{l}\text { 5-year survival } \\
\text { rate }\end{array}$ \\
\hline Alaska & 122 & 68.33 & 50.19 & 0.42 & 0.26 & 0.19 & 0.16 & 0.13 \\
\hline California & 25,225 & 64.41 & 49.44 & 0.40 & 0.23 & 0.17 & 0.14 & 0.13 \\
\hline Connecticut & 6832 & 65.74 & 48.40 & 0.41 & 0.26 & 0.19 & 0.16 & 0.14 \\
\hline Georgia & 7832 & 67.88 & 45.13 & 0.48 & 0.31 & 0.24 & 0.20 & 0.17 \\
\hline Hawaii & 1669 & 65.80 & 48.69 & 0.40 & 0.23 & 0.16 & 0.13 & 0.12 \\
\hline lowa & 4983 & 67.86 & 51.87 & 0.42 & 0.26 & 0.20 & 0.16 & 0.15 \\
\hline Kentucky & 3484 & 68.03 & 50.49 & 0.43 & 0.27 & 0.21 & 0.18 & 0.16 \\
\hline Louisiana & 3394 & 65.90 & 47.01 & 0.42 & 0.27 & 0.22 & 0.18 & 0.17 \\
\hline Michigan & 7624 & 65.07 & 48.31 & 0.44 & 0.27 & 0.21 & 0.17 & 0.15 \\
\hline New Jersey & 6762 & 67.49 & 53.91 & 0.39 & 0.22 & 0.17 & 0.13 & 0.11 \\
\hline $\begin{array}{l}\text { New } \\
\text { Mexico }\end{array}$ & 1892 & 67.58 & 47.70 & 0.42 & 0.24 & 0.17 & 0.14 & 0.12 \\
\hline Utah & 1503 & 67.27 & 55.90 & 0.34 & 0.18 & 0.13 & 0.10 & 0.08 \\
\hline Washington & 5951 & 64.18 & 55.43 & 0.44 & 0.28 & 0.23 & 0.22 & 0.22 \\
\hline
\end{tabular}


not make full use of the comprehensive information in SEER. ECCDIA makes the most use of EC data of the SEER database and presents these data in a user-friendly interactive interface with no need to grasp computational programming skills. It can easily exhibit the clinicopathologic and prognostic analysis for a variety of subgroups of EC patients.

To the best of our knowledge, the online tool ECCDIA is the first such system that demonstrates the most comprehensive integrative analysis of clinical data with the full utilization of EC data in the SEER database. More importantly, to facilitate clinical use of this online tool, nomograms predicting the prognosis of different subgroups of EC patients are provided are provided by ECCDIA. Using ECCDIA, clinicians can immediately obtain the survival probability of patients by simply inputting the values of clinical factors, which helps them make the right decision for EC patients.

There are already many good online tools for esophageal cancer. For example, both OSescc and OSeac are great tools that use gene expression data of esophageal cancer patients in public databases to quickly query the correlation between the expression level of a gene and patient prognosis $[15,16]$. Briefly, compared with OSescc and or OSeac, ECCDIA is committed to creating dynamic interactive visualization tools to explore the epidemiological characteristics of esophageal cancer in the SEER database. In addition to survival analysis, ECCDIA can also dynamically and interactively display the epidemiological characteristics of esophageal cancer patients spanning 20 years. Both gene expression data and epidemiological characteristics can provide complementary information for better understanding esophageal cancer.

Some limitations of ECCDIA need to be mentioned. ECCDIA does not integrate the molecular or genetic data of EC patients with their clinical data, since the SEER database only provides the clinical data of cancer patients. In addition, some treatment biases are present. Therefore, additional future work is needed by combining the data in the SEER database with other publicly available databases.

Nevertheless, ECCDIA is the first interactive web tool to assess the largest clinical and prognostic data of EC patients, which will become an invaluable resource for clinical guideline of EC. Besides, ECCDIA will be updated to embrace the newest data released by SEER.

\section{Conclusion}

The Esophageal Cancer Clinical Data Interactive Analysis (ECCDIA, http://webapps.3steps.cn/ECCDIA/) is the first interactive prediction and visualization web tool to assess the largest clinical and prognostic data of EC patients from the SEER database, further increasing the assessment of clinical guidelines for EC. Furthermore, ECCDIA will be regularly updated to embrace the newest data to be released by SEER.

\section{Supplementary information}

Supplementary information accompanies this paper at https://doi.org/10. 1186/s12885-020-07479-9.

Additional file 1: Figure S1. Histologic type ratio distribution by year. Figure S2. The patient flows between histologic type and race. Figure S3. Survival analysis example. Figure S4. The relationship between histologic type and survival. Figure S5. Survival rate prediction. Figure S6. Survival prediction external verification.

\section{Abbreviations}

EC: Esophageal cancer; SEER: The Surveillance, Epidemiology, and End Results; KM: Kaplan-Meier; ECCDIA: Esophageal Cancer Clinical Data Interactive Analysis; ICD-O-3: International Classification of Diseases for Oncology; ESCC: Esophageal squamous cell carcinoma; EAD: Esophageal adenocarcinoma; OS: Overall survival; CSS: Cancer specific death

\section{Acknowledgements}

The authors would like to thank The Genius Medicine Consortium (TGMC) for providing technical support.

\section{Authors' contributions}

JY and JS designed the study. JS, ZY and QS collected the data and performed the statistical analysis. JY and JS developed the software. JY, QS, $Z Y, J C, Y Y$ and LS wrote and revised the paper. All authors read and approved the final manuscript.

\section{Funding}

This work was supported in part by the National Key R\&D Project of China (2018YFE0201600, 2017YFC0907502, and 2017YFF0204600), the National Natural Science Foundation of China (31720103909), and Shanghai Municipal Science and Technology Major Project (2017SHZDZX01) and the 111 Project (B13016). Funding for open access charge: National Key R\&D Project of China [2018YFE0201600].

\section{Availability of data and materials}

The datasets generated and/or analyzed during the current study are available in the clinico-omics/ECCDIA repository, https://github.com/clinicoomics/ECCDIA.

Ethics approval and consent to participate

Not applicable.

Consent for publication

Not applicable.

Competing interests

The authors declare that they have no competing interests.

\section{Author details}

${ }^{1}$ State Key Laboratory of Genetic Engineering, School of Life Sciences and Shanghai Cancer Hospital/Cancer Institute, Fudan University, Shanghai 200438, China. ${ }^{2}$ Department of Hematology and Oncology, The Affiliated Hospital of Hangzhou Normal University, Hangzhou 310015, Zhejiang, China. ${ }^{3}$ Medical College of Soochow University, Suzhou 215000, Jiangsu, China. ${ }^{4}$ Human Phenome Institute, Fudan University, Shanghai 201203, China.

${ }^{5}$ Fudan-Gospel Joint Research Center for Precision Medicine, Fudan University, Shanghai 200438, China. 
Received: 12 February 2020 Accepted: 1 October 2020

Published online: 12 October 2020

\section{References}

1. Siegel RL, Miller KD, Jemal A. Cancer statistics, 2019. CA Cancer J Clin. 2019 Jan;69(1):7-34

2. Bray F, Ferlay J, Soerjomataram I, Siegel RL, Torre LA, Jemal A. Global cancer statistics 2018: GLOBOCAN estimates of incidence and mortality worldwide for 36 cancers in 185 countries. CA Cancer J Clin. 2018;68(6):394-424 American Cancer Society.

3. Baba Y, Yoshida N, Kinoshita K, Iwatsuki M, Yamashita Y-I, Chikamoto A, et al. Clinical and prognostic features of patients with esophageal cancer and multiple primary cancers: a retrospective single-institution study. Ann Surg. 2018 Mar 1;267(3):478-83.

4. Napier KJ. Esophageal cancer: a review of epidemiology, pathogenesis, staging workup and treatment modalities. WJGO. 2014;6(5):112-0 Baishideng Publishing Group Inc.

5. Bohanes P, Yang D, Chhibar RS, Labonte MJ, Winder T, Ning Y, et al. Influence of sex on the survival of patients with esophageal cancer. J Clin Oncol. 2012;30(18):2265-72.

6. Njei B, McCarty TR, Birk JW. Trends in esophageal cancer survival in United States adults from 1973 to 2009: a SEER database analysis. J Gastroenterol Hepatol. 2016;31(6):1141-6 3rd ed. John Wiley \&amp; Sons, Ltd (10.1111).

7. Benson K, Hartz AJ. A comparison of observational studies and randomized, controlled trials. N Engl J Med. 2000;342(25):1878-86.

8. Doll KM, Rademaker A, Sosa JA. Practical guide to surgical data sets: surveillance, epidemiology, and end results (SEER) database. JAMA Surg. 2018;153(6):588-9.

9. SEER*Explorer: An interactive website for SEER cancer statistics [Internet] Surveillance Research Program, National Cancer Institute. Available from https://seer.cancer.gov/explorer/. [Cited 2018 Nov 14].

10. Berry MF, Zeyer-Brunner J, Castleberry AW, Martin JT, Gloor B, Pietrobon R, et al. Treatment modalities for T1N0 esophageal cancers: a comparative analysis of local therapy versus surgical resection. J Thorac Oncol. 2013;8(6): 796-802.

11. Sievert C, Parmer C, Hocking T, Chamberlain S, Ram K. Plotly: create interactive web graphics via plotly's javascript graphing library; 2016.

12. Therneau TM, Grambsch PM. Modeling Survival Data: Extending the Cox Model. New York: Springer. ISBN 0-387-98784-3.

13. Kassambara A, Kosinski A, Biecek P, 0.4 SFRPV. Survminer: drawing survival curves using ggplot2. 2019.

14. Harrel FE Jr. rms: regression modeling strategies. R package version 5.1-2; 2018.

15. Wang Q, Wang F, Lv J, Xin J, Xie L, Zhu W, Tang Y, Li Y, Zhao X, Wang Y, Li $X$, Guo $X$. Interactive online consensus survival tool for esophageal squamous cell carcinoma prognosis analysis. Oncol Lett. 2019;18(2):1199_ 206.

16. Wang Q, Yan Z, Ge L, Li N, Yang M, Sun X, Xie L, Zhang G, Zhu W, Wang Y, Li Y, Li X, Guo X. OSeac: an online survival analysis tool for esophageal adenocarcinoma. Front Oncol. 2020;10:315.

\section{Publisher's Note}

Springer Nature remains neutral with regard to jurisdictional claims in published maps and institutional affiliations.

Ready to submit your research? Choose BMC and benefit from:

- fast, convenient online submission

- thorough peer review by experienced researchers in your field

- rapid publication on acceptance

- support for research data, including large and complex data types

- gold Open Access which fosters wider collaboration and increased citations

- maximum visibility for your research: over $100 \mathrm{M}$ website views per year

At $\mathrm{BMC}$, research is always in progress.

Learn more biomedcentral.com/submissions 